\title{
Stable Genetic Adaptive Control by Using a 2-DOF Control Scheme
}

\author{
Asier Ibeas*
}

\author{
Depto. de Telecomunicaciones e Ingeniería de Sistemas, Escuela Técnica Superior de Ingeniería. Universidad \\ Autónoma de Barcelona, 08193 Cerdanyola del Vallès (Bellaterra). Barcelona. Spain
}

\begin{abstract}
This paper introduces an adaptive controller based on the online genetic estimation of the parameters of the system. The main novelty of the paper relies on the fact that the stability of the genetic adaptive scheme is fully tackled proving the boundedness of all systems signals under weak conditions on the genetic population evolution. Thus, the proposed set-up is flexible enough to be integrated within a great variety of genetic estimation algorithms. The goal is achieved by using a certain Two-Degree-of-Freedom (2-DOF) based implementation of the control law. The procedure for the genetic controller synthesis is described while its properties and requirements are stated.
\end{abstract}

Keywords: Genetic controllers, stability, adaptive control, 2-DOF schemes.

\section{INTRODUCTION}

Genetic Algorithms (GAs) have been revealed as powerful tools for solving a wide variety of problems since their introduction by Holland in 1975 [1]. Basically, they act as optimization tools able to tackle complex problems which are practically intractable from an alternative (analytically) point of view. Several areas in Science and Engineering have benefited from its approach such as Economics, Structural Design, Network Design, Game Theory and Operations Research for citing few of them [2]. Moreover, the GA has also found application in the area of automatic control systems design [3]. Typically, for this, the controller is formulated by using a set of parameters which the GA tries to optimize in some sense by using simulation of the potential operation of each set of candidate values in the closed-loop scheme. GA based approaches to controller design can be condensed into three major categories.

i) The first one consists in integrating GAs with fuzzy or neuro-fuzzy controllers with the aim at improving (or decreasing) the set of rules necessary to design the fuzzy subsystem (see, for instance [4-6]).

ii) The second one consists in using the GA in the offline optimization of the controller or observer composing the closed-loop [7]. There are several works covering this topic, including the observer design [2], state feedback control laws [8,9], PID control [7,10] and $H_{\infty}$ optimization [3].

iii) Finally, the third approach consists in the use of GAs to the online (and, hence, time-varying) determination of the controller. Thus, this approach fits in well with the adaptive control frame.

*Address correspondence to this author at the Depto. de Telecomunicaciones e Ingeniería de Sistemas, Escuela Técnica Superior de Ingeniería. Universidad Autónoma de Barcelona, 08193 Cerdanyola del Vallès (Bellaterra). Barcelona. Spain; E-mail: Asier.Ibeas@uab.es
There are several works pointing out the advantages of using GA in the online estimation of the controller parameters [10-13]. Reported advantages include the fast convergence to adequate controller parameters $[12,14]$, direct identification of poles and zeros instead of plant coefficients and improved robustness properties in comparison with traditional recursive estimation schemes $[10,12,15]$. The conclusion of these works is that GAs are powerful tools to be included within an adaptive control framework.

Nevertheless, most of these works (even the most recent ones) do not tackle explicitly the stability issue in their development [12]. Main reason for this relies on the fact that GAs are heuristic algorithms whose evolution is basically dictated by probabilistic rules which makes difficult to predict the evolution of the controller parameters used to implement the control law. Hence, works covering the genetic adaptive control of systems do not usually include the stability analysis of the resulting closed-loop but just a demonstration, through simulation or experimental work, of the advantages provided by this kind of controllers [10,12]. Furthermore, those exploring the stability issue usually include a number of probabilistic-type hypotheses on the parameter evolution in order to guarantee the closed-loop stability (see, for instance [11]).

In this paper, a Two Degree of Freedom (2-DOF) approach to implement genetic adaptive control laws is proposed. The main interest of the proposed approach relies on the fact that main advantages of the genetic estimation can be taken into account when designing the scheme while the stability of the closed-loop system can be explicitly tackled and guaranteed. Indeed, the stability of the closed-loop is proved under weak feasible conditions on the parameter evolution. This benefit is obtained by using a certain implementation of a 2-DOF control law introduced in $[16,17]$. In this way, stability is mainly guaranteed by an internal fixed feedback controller while the tracking performance is achieved by genetically modified external compensators. Thus, the stability of the complete scheme is displaced to the internal 
compensator being weakly influenced by the evolution of the genetic population. Hence, basic advantages of GA can be linked with guaranteed stability properties.

The paper is organised as follows. Section 2 introduces the problem formulation and main objectives. In Section 3, the genetic design of the controller is commented. Section 4 states the stability properties of the resulting closed-loop system. Section 5 is devoted to simulation examples while conclusions end the paper.

\section{PROBLEM FORMULATION}

We consider the problem of controlling a LTI continuous-time system described by:

$$
G(s)=\frac{M(s)}{N(s)}
$$

where $M(s)$ and $N(s)$ are polynomials in the Laplace variable $s$, with unknown parameters, satisfying the following assumption.

Assumption 1. Upper-bounds for the plant polynomial degrees are known:

$\operatorname{deg} N(s) \leq \bar{n}, \operatorname{deg} M(s) \leq \bar{m}$

with $\bar{n} \geq \bar{m} \geq 0$ known.

Thus, we are considering the nominal model affected of parametric uncertainty while no unmodeled dynamics are taken into account.

Since it is very common nowadays to control in practical engineering a continuous-time process using a discrete-time controller, we will consider a discrete model of the plant in order to synthesize a discrete-time controller. Therefore, the design procedure proposed in this paper will apply to both discretized continuous systems and to purely discrete ones just avoiding the previous discretization step. This setup is preferred rather than an original continuous-time one in order to incorporate into a theoretical framework the way in which control systems are usually now implemented in practice.

\subsection{Discrete Plant Model}

A discrete model of (1) is obtained using a zero order hold $(\mathrm{ZOH})$ with sampling period $T_{s}$ according to:

$$
\begin{aligned}
& Z\left[\frac{1-e^{-s T_{s}}}{s} G(s)\right]=\left(1-z^{-1}\right) Z\left[\frac{G(s)}{s}\right] \\
& =\left(1-z^{-1}\right) Z\left[\frac{M(s)}{s N(s)}\right]=\left(1-z^{-1}\right) \frac{N_{p}^{\prime}\left(z^{-1}\right)}{D_{p}^{\prime}\left(z^{-1}\right)}=\frac{N_{p}(z)}{D_{p}(z)}
\end{aligned}
$$

with

$$
\begin{aligned}
& N_{p}(z)=z^{\bar{n}}+a_{\bar{n}-1} z^{\bar{n}-1}+\ldots+a_{0} \\
& D_{p}(z)=b_{\bar{m}} z^{\bar{m}}+b_{\bar{m}-1} z^{\bar{m}-1}+\ldots+b_{0}
\end{aligned}
$$

where $Z$ denotes the zeta-transform and $z^{-1} \leftrightarrow q^{-1}$ (while $z \leftrightarrow q$ ) is equivalent to the one sampling period backward operator (one sample period ahead operator). Moreover, upon Assumption 1 holds, the following degree relations appear in the discrete plant (2):

$\operatorname{deg} D_{p}(z) \leq \bar{n}, \operatorname{deg} N_{p}(z) \leq \bar{m}$

since a $\mathrm{ZOH}$ has been used to obtain the corresponding discrete-time model [18]. The knowledge of these orders allows specifying the size of the chromosomes in the GA formulation. Hence, the original problem is reformulated into the control of a discrete-time linear system with parametric uncertainty. Thus, since the plant parameters are unknown a genetic estimation algorithm will be used to estimate the value of the polynomials $N_{p}(z), D_{p}(z)$ converting them into time-varying, denoted $\hat{N}_{p k}(z), \hat{D}_{p k}(z)$. The GA will be used to obtain such estimated polynomials in such a way that its use within an online adaptive control scheme results into an asymptotically stable closed-loop system. Nevertheless, its utilization within an adaptive controller usually lacks from stability considerations as commented in Section 1. In the sequel, an implementation of a 2-DOF control law allows linking the advantages of the genetic estimation along with guaranteeing stability properties under weak conditions on the evolution of the genetic population which is the main contribution of the paper.

\section{CONTROLLER DESIGN PROCEDURE}

This Section describes the control scheme proposed to assess the stability within a genetic adaptive estimation scheme framework for the plant with parametric uncertainty (1) through its discrete-time counterpart (2). Firstly, the basic topology of the control scheme, is introduced in the following section.

\subsection{Two Degree of Freedom (2-DOF) Control Law Im- plementation}

In this section we concentrate on the basic 2-DOF control law implementation introduced in $[16,17]$ and displayed in Fig. (1) for convenience.

There are several implementations of the general two degree of freedom controller. However, as pointed out in [19], not all of them possess the same closed-loop properties. Thus, the above topology is the most adequate to be used within the genetic algorithm context. Within this configuration, the transfer function from the reference $r_{k}$ to the output $y_{k}$ is computed to be (dropping $z$ hereafter for the sake of simplicity):

$$
T_{y r}=N_{p} C\left(\frac{D_{p}+C_{0} N_{p}}{D_{p}+C_{0} N_{p}}\right)=N_{p} C\left(\frac{R D_{p}+S N_{p}}{R D_{p}+S N_{p}}\right)
$$




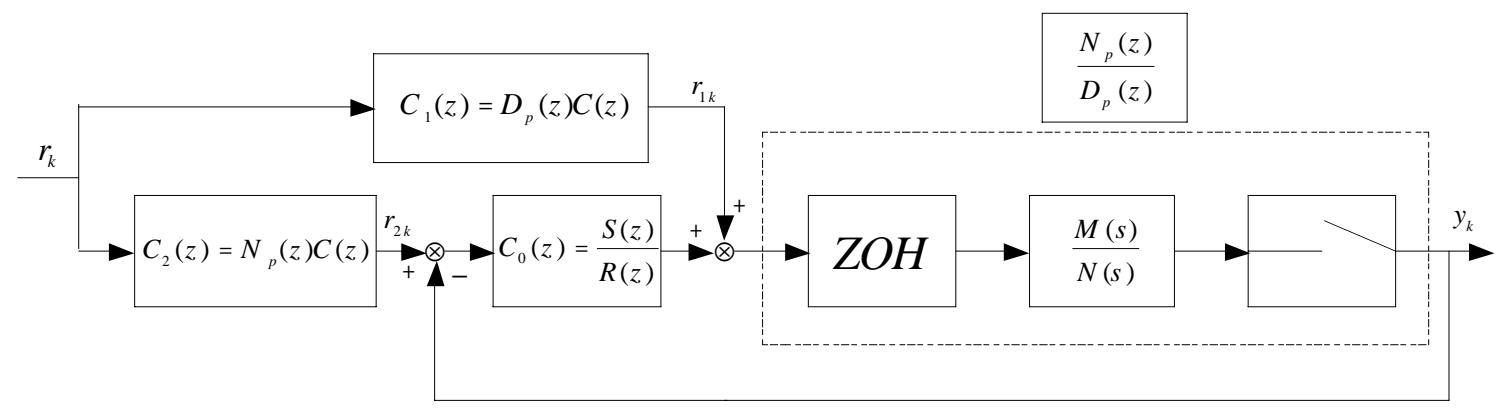

Fig. (1). 2-DOF control law implementation.

for a feedback controller realization $C_{0}=S / R$ where a pole-zero polynomial cancellation has been explicitly indicated in the closed-loop calculation in order to show the role played by each compensator in the closed-loop properties. Thus, the pole-zero cancellation can be performed in (4) when $R D_{p}+S N_{p}$ is Schur. Note that this polynomial represents the dynamic equation of the closed-loop system appearing when a single degree of freedom configuration (i.e., setting $C_{1}=0 ; C_{2}=1$ in Fig. (1)) is used. Hence, the compensator $C_{0}$ can be designed to stabilize the system as it were the unique controller in the closed-loop (stabilising compensator). Having stabilized the inner-loop, the polezero polynomial cancellation in (4) can be finally performed leading to an output only conditioned (in the steady-state) by the external transfer function $N_{p} C$ according to:

$y_{k}=\mathbf{Z}^{-1}\left[N_{p} C r\right]+v_{k}$

where $v_{k}$ is an exponentially vanishing signal associated with potential non-zero initial conditions of the cancelled poles. Hence, the steady-state output of the closed-loop is determined by the choice of the compensator $C$ in $N_{p} C$ regardless the value of $C_{0}$ stabilizing the inner-loop. Thus, within this control configuration, both controllers can be designed according to a differentiate design criteria, pointing out their independence during the controller design process. Therefore, the problem is decomposed into the design of a 1DOF robust stabilising compensator, followed by the design of a tandem of external compensators which are aimed at selecting the behaviour of the output. The above scheme is enhanced when parametric uncertainty in the nominal model is taken into account in the following Section.

\subsection{2-DOF Control Topology in the Presence of Para- metric Model Uncertainty}

If a nominal model of the plant, denoted by $\hat{N}_{p}, \hat{D}_{p}$, is known instead of the actual plant $N_{p}, D_{p}$, the original closed-loop control diagram (Fig. 1) becomes:

which implies a closed-loop transfer function

$T_{y r}=N_{p} C\left(\frac{R \hat{D}_{p}+S \hat{N}_{p}}{R D_{p}+S N_{p}}\right)$

Therefore, in the case when a parametric mismatch between the plant model and the actual plant is taken into account, the above separation principle is no longer valid since the pole-zero polynomial cancellation in (4) cannot be performed in (6). Hence, the closed-loop response is impacted (and, in general, degraded) by the poles of the inner control loop.

Moreover, it can be concluded from (6) that the compensator $C_{0}$ is still playing the same role as before: it has to stabilize the inner loop but now, the performance is associated to all compensators $C_{0}, C_{1}$ and $C_{2}$ (involving $\hat{D}_{p}$ and $\hat{N}_{p}$ respectively) losing the differentiate design criteria.

Nevertheless, by converting the external compensators into adaptive, we will be able to correct the (potential) mismatch between the nominal model and the real system pro-

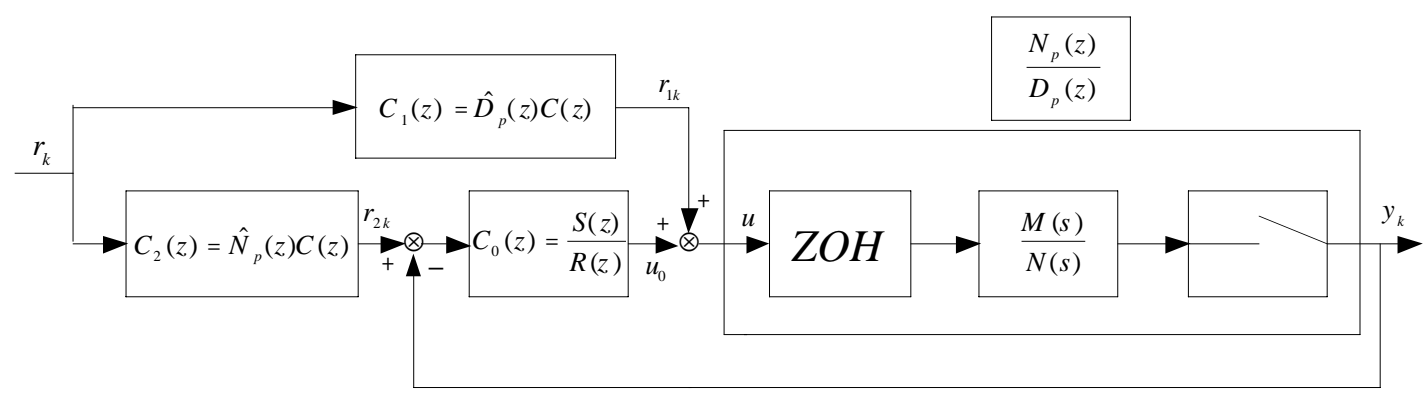

Fig. (2). 2-DOF control scheme in the presence of uncertainty. 
viding the approximate pole-zero cancellation in (6) achieving a certain level of performance of the closed-loop while the stability is (mainly) guaranteed by the internal compensator.

In particular, we will consider the scenario where a stabilizing fixed (time-invariant) controller $C_{0}$ is obtained somehow from the nominal plant model $\hat{N}_{p}, \hat{D}_{p}$ while converting the external compensators $C_{1}, C_{2}$ into timevarying $C_{1 k}, C_{2 k}$. Thus, the following structure is considered for the external compensators obtained by setting the values of the estimated polynomials $\hat{N}_{p k}, \hat{D}_{p k}$ in $C_{1 k}, C_{2 k}$ while maintaining the same transfer function $C$ in Fig. (2):

$C_{1 k}=\hat{D}_{p k} C, C_{2 k}=\hat{N}_{p k} C$

The polynomials $\hat{N}_{p k}, \hat{D}_{p k}$ are to be estimated taking the advantages of the GAs. In this way, the stability of the closed-loop is achieved by the internal feedback compensator while the genetic estimation is to be used to update the out-of-the-loop ones through the plant identified polynomials. Thus, a stable adaptive scheme able to achieve adequate closed-loop performance is proposed while incorporating the advantages of a genetic estimation. Hence, within this control configuration, the stability problem is displaced to the design of the stabilizing controller while the advantages of the genetic estimation are used in the external compensators design. Thus the stability problem is separated from the design of the genetic algorithm leading to a modular way to design the genetic control law.

Note that, in this scenario, we do not worry about the design of the internal controller: it is obtained using any of the robust design procedures proposed in the literature for 1DOF control configurations (see, for instance [20,21]). The unique requirement on $C_{0}$ is that it has to stabilize the inner-loop system. Thus, the following Assumption is made in order to carry out the stability proof:

Assumption 2. The fixed feedback controller $C_{0}$ stabilizes the actual inner loop.

\subsection{Genetic Estimation of the Plant}

The genetic estimation of the plant acts modifying the external compensators. In order to subsequently state the stability of the closed-loop with time-varying external subsystems, the following slight technical assumption on the GA population evolution is made:

Assumption 3. The population of the genetic algorithm is bounded at all time.

Remarks.

1. This boundedness property is typical in many genetic algorithms used in identification (see, for instance [10,12]), and hence, is a very slight assumption on applications of GAs to control.
The above assumption is needed to guarantee closed-loop stability and enables the use of any genetic algorithm proposed in the literature satisfying Assumption 3 to perform the identification process. This makes the proposed setup of great flexibility and interest. However, in order to show some simulation examples showing the usefulness of the proposed scheme, the following genetic estimation algorithm is proposed, following the steps given in [14].

\subsection{Genetic Estimation Algorithm}

This Section contains an estimation algorithm inspired from GAs. Its main purpose relies on the fact of showing, in examples, the behaviour of the proposed 2-DOF scheme. Thus, in the following, a description of the algorithm is stated without proving that Assumption 3 is satisfied while testing it through simulation examples.

\subsubsection{Codification}

Chromosomes (individuals) will be real-valued vectors with size $(\bar{n}+\bar{m}+1)$ from Assumption 1. The chromosomes will be denoted by $\theta_{k}^{(i)}$ at each sampling time $t=k T_{s}, k \in \mathbb{N}$ for $i=1,2, \ldots, n_{c}$, where $n_{c}$ is the total number of chromosomes (or size of the population). These vectors are selected in such a way that the corresponding output of the plant could be written for each chromosome as:

$y_{k}^{(i)}=\varphi_{k}^{T} \theta_{k}^{(i)}$

$\varphi_{k}^{T}=\left[\begin{array}{llllllll}-y_{k-1} & -y_{k-2} & \cdots & -y_{k-\bar{n}} & u_{k-\bar{n}+\bar{m}} & u_{k-\bar{n}+\bar{m}-1} & \cdots & u_{k-\bar{n}}\end{array}\right]$

$\theta_{k}^{(i)}=\left[\begin{array}{llllllll}a_{\bar{n}} & a_{\bar{n}-1} & \cdots & a_{0} & b_{\bar{m}} & b_{\bar{m}-1} & \cdots & b_{0}\end{array}\right]$

where $\varphi_{k}$ is the so called regressor, containing input-output measured data form the system. In other words, chromosomes are selected to fit in with the typical inner product realization of the discrete-time plant proposed, for instance, in [22].

\subsubsection{Fitness Evaluation}

The fitness of each individual is evaluated using the deviation of each potential chromosome output (8) from the actual output of the plant $y_{k}$. In particular, the fitness measure at time $t=k T_{s}, k \in \mathbb{N}$ is:

$J_{k}^{(i)}=\frac{1}{\left(y_{k}-y_{k}^{(i)}\right)^{2}+\varepsilon} ; i=1,2, \ldots, n_{c}$

where $J_{k}^{(i)}$ denotes the fitness of individual $\theta_{k}^{(i)}$ and $\varepsilon>0$ is a small positive offset used to guarantee the wellposedness of (9). Note that the best individuals are those with the largest fitness (Fig. 9), since those reveal a closer behaviour of them to the behaviour of the actual system. In [14], interested reader can find a number of alternative fitness functions. 


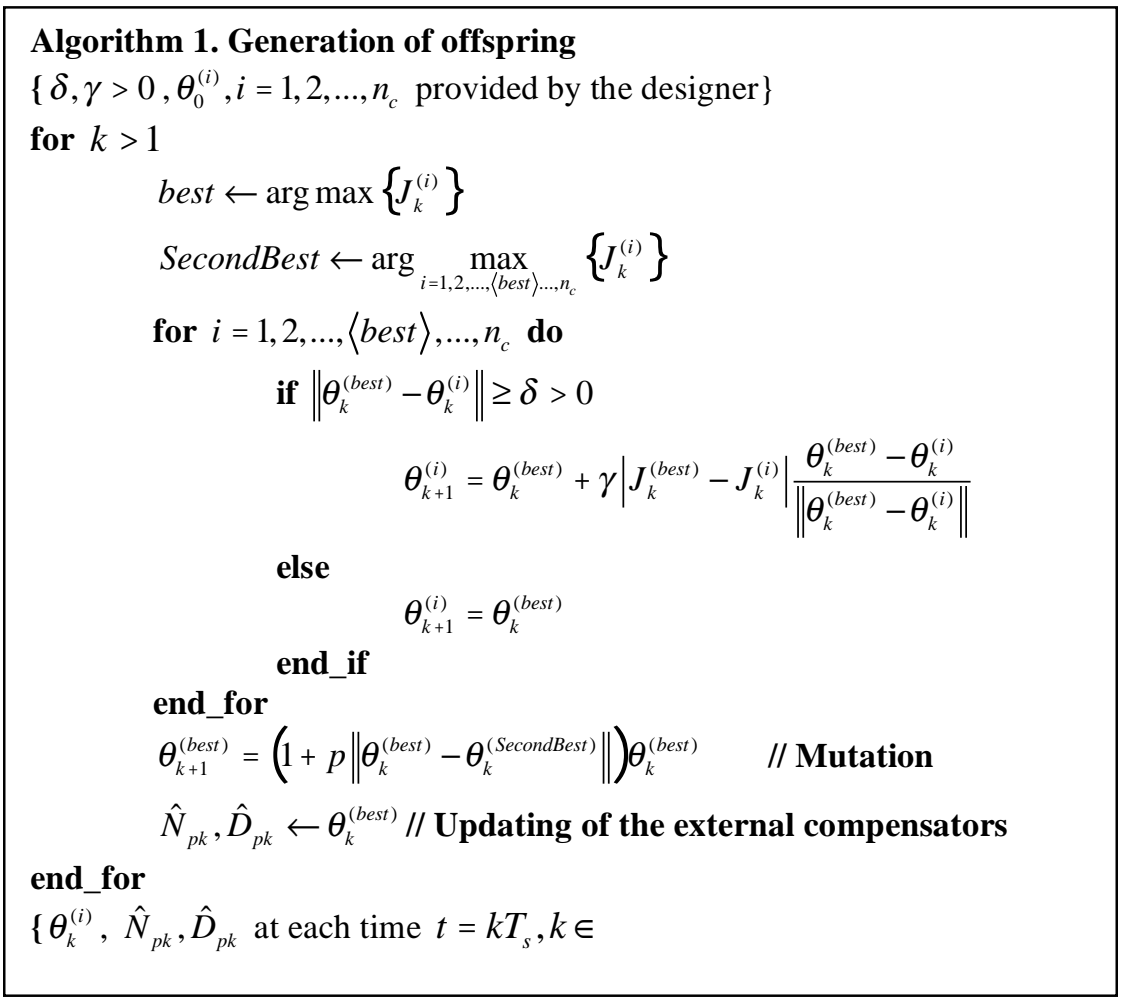

\subsubsection{Offspring Generation and Mutation}

The new individuals for the next generation (offspring) are obtained from the previous generation (parents) using the algorithm 1, where $\langle$ best $\rangle$ denotes that the element labelled as 'best' has been removed from the 'for' loop and $p$ is a random number belonging to $[-1,1]$.

The algorithm works as follows. The designer initializes the tentative chromosomes either in a random way or taking into account the available 'a priori' information on the system. Then, at each sampling time, the potential set of plant parameters are evaluated according to their fitness evaluation index (9). Then, the best individual is selected from the set of potential candidates. This element is used to parameterize the external compensators at time $t=k T_{s}, k \in$. Moreover, from the original set of individuals, the offspring is obtained by guiding the remaining elements in the direction of the best one with a gain proportional to the weighted distance from the original individual to the best one. The weight is proportional to the difference in their evaluation functions. The best individual is not modified in this step. This process is schematically described in Fig. (3).

Finally, as Fig. (4) below shows, the best individual is randomly mutated with amplitude proportional to its distance to the second best vector. Convergence properties are not analytically stated, but simulation examples will show that population finally converges to a finite fixed value of the estimated parameters as required by Assumption 3 .

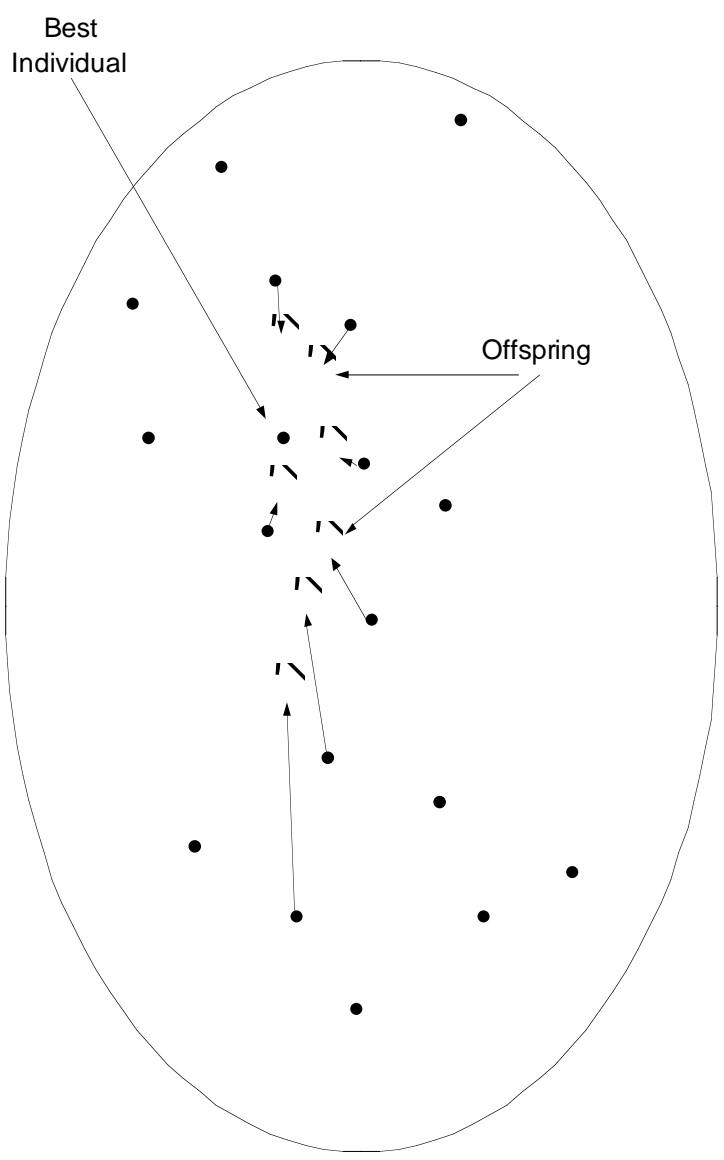

Fig. (3). Generation of the offspring. 


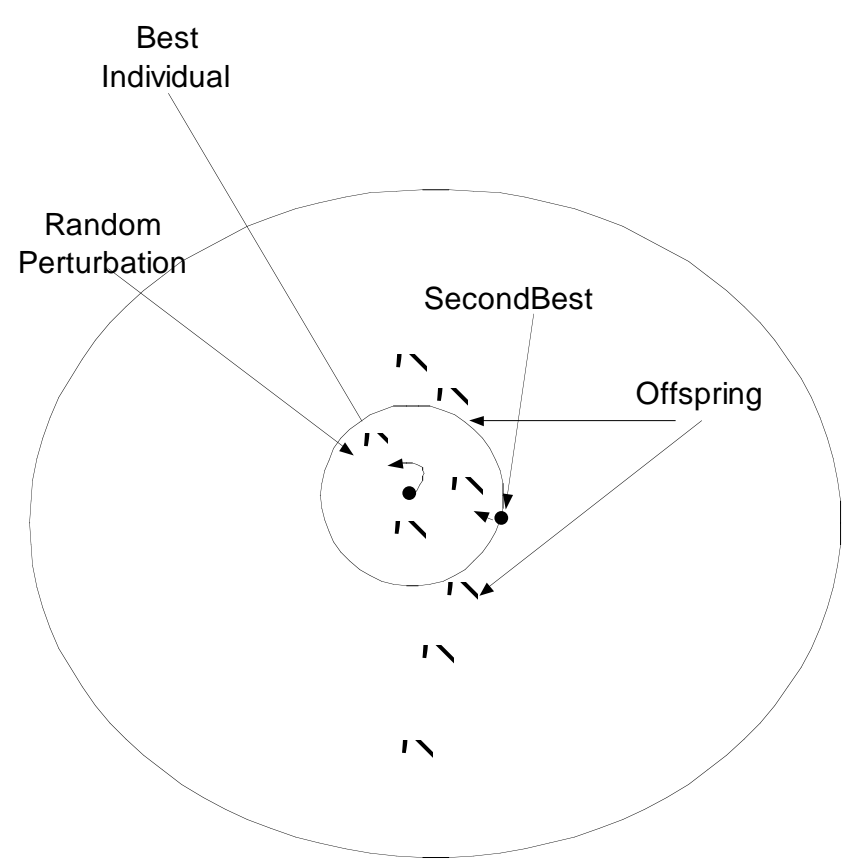

Fig. (4). Random perturbation (mutation) of the best individual.

\subsection{Control Law}

The discrete-time control law composed of the sequence of control inputs $\left\{u_{k}\right\}_{k=0}^{\infty}$ is generated according to the diagram shown in Fig. (2) by compensators $C_{0}, C_{1 k}, C_{2 k}$. In order to guarantee the adequate behaviour of the scheme, the following feasible technical assumptions are considered.

Assumption 4. The reference signal $r$ is bounded.

Furthermore, since the external controller $C$ determines the behaviour of the closed-loop response (4),(6) it must satisfy the following assumption to guarantee closed-loop stability.

Assumption 5. The compensator $C$ is strictly stable.

There are several ways to generate the control law depending on the design of the compensators. Interested reader can consult, for instance [16,22]. Finally, the continuoustime control law is obtained by using a zero-order hold:

$u(t)=u_{k}, t \in\left[k T_{s},(k+1) T_{s}\right)$

\section{CLOSED-LOOP STABILITY}

With the above definitions for the plant and control law implementation, we are able to state the stability result.

Theorem 1. The feedback control system for the plant (1) through its discretized model (2) described in Fig. (2) with compensators satisfying Assumptions (2),(5) is globally stable provided that genetic estimation and reference signal satisfy Assumptions (3),(4).

The proof of this theorem is contained in Appendix I. In particular, in order to use the above stability result, the evolution of the population of the GA has to be bounded at all time. The key property to asses the stability of the closedloop despite any probabilistic GA (satisfying Assumption 3) being used is the particular implementation of the control law which allows displacing the stability of the system to the internal controller while using the genetic estimation algorithm, with all his advantages, in the design of the external compensators.

The proof is organised into three steps:

1. The output of the first compensator $C_{1}$ is proved to be bounded provided Assumptions 3, 4 and 5 hold.

2. Similarly, the output of the second compensator $C_{2}$ is proved to be bounded provided Assumptions 3, 4 and 5 hold.

3. Finally, the closed-loop system is written as the internal-loop with two external bounded input signals, namely, $r_{1}$ and $r_{2}$ as shown in the following Fig. (5).

Hence, the stability is deduced from the corresponding closed-loop calculation provided that Assumption 2 holds by using the (previous proved) facts that $r_{1}$ and $r_{2}$ are bounded. Technical details are contained in Appendix I. Furthermore, in the following Section a simulation example showing the usefulness of the proposed approach is presented.

\section{SIMULATION EXAMPLES}

This section contains some simulation examples concerning the genetic estimation algorithm described in the previous Section 3. The system to be controlled is the discretetime plant:

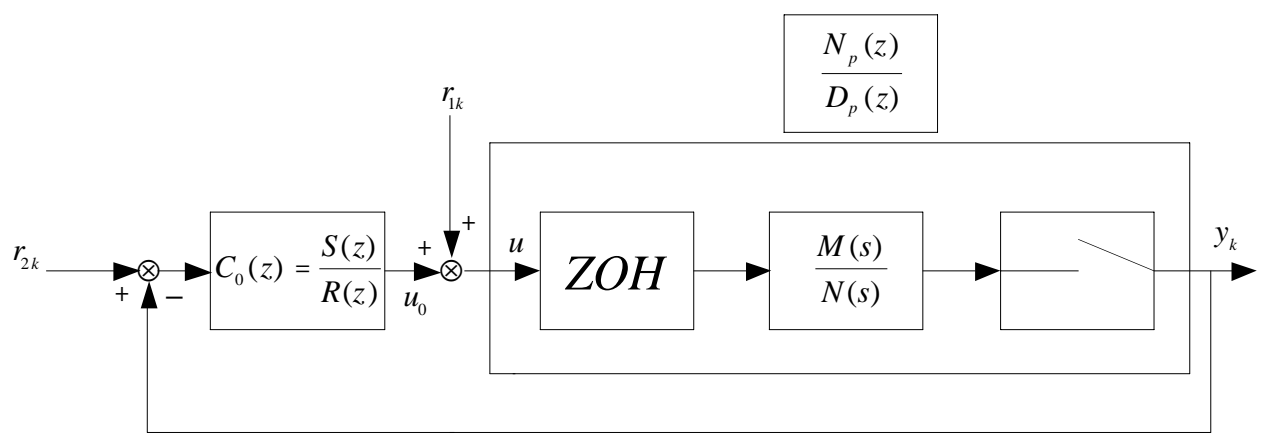

Fig. (5). System with external signals. 


$$
H(z)=\frac{z^{2}-0.6 z+0.0875}{z^{3}+1.9 z^{2}-0.195 z+0.73}
$$

with associate parameter vector

$$
\theta^{T}=\left[\begin{array}{lll}
\theta_{1} & \cdots & \theta_{6}
\end{array}\right]=\left[\begin{array}{llllll}
-1.9 & 0.73 & -0.195 & 1 & -0.6 & 0.0875
\end{array}\right]
$$

The population is composed of 250 individuals randomly initialized, while the updating of the offspring is done by the algorithm proposed in the previous Section 3. The internal controller used to stabilize the inner loop (and, hence, satisfying Assumption 2) is selected to be:

$$
C_{0}(z)=\frac{1.4286 z^{2}-0.5714 z+0.1429}{z^{2}-0.4286 z+0.0857}
$$

The reference signal is

$$
r(t)=\sin (4 \pi t)+3 \sin (6 \pi t)+2 \sin (8 \pi t)
$$

with a sampling period of $T_{s}=0.02 \mathrm{sec}$. The following simulations are obtained for the output of the control scheme (Fig. 6) and the evolution of the parameters of the best individual (Fig. 7):

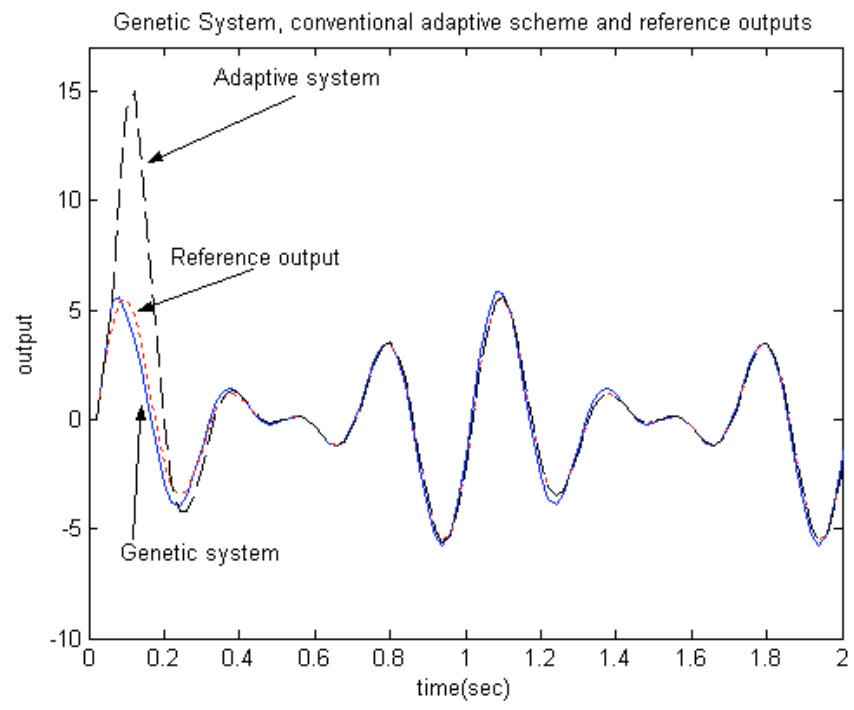

Fig. (6). Comparison of outputs between genetic 2-DOF scheme and conventional adaptive one.

As shown in Fig. (6), the output of the system with the genetic estimation improves the use of a single traditional estimation algorithm tracking the non-constant reference signal stated below. This property is not surprising due to the relationship between multi-estimation controllers and GA pointed out, for instance, by the authors in [14]. Moreover, Fig. (6) shows the appropriate working of the control system with the genetic estimation introduced in Section 3.3 where the estimation of the parameters of the system converges to a finite limit. Furthermore, Fig. (7) shows, as an example, the boundedness of the best estimator, leading to adequate closed-loop performances as shown in Fig. (6).

\section{CONCLUSIONS}

In this paper, a genetic adaptive control scheme capable of guaranteeing closed-loop stability is proposed. The

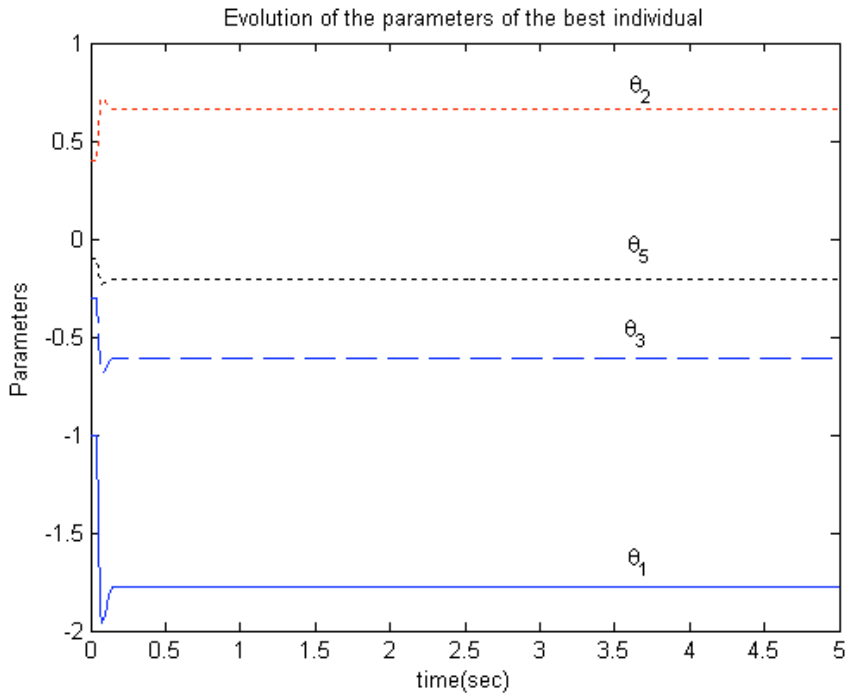

Fig. (7). Evolution of the parameters of the best individual.

control law is based on a 2-DOF scheme which displaces the stability requirements to an internal stabilizing compensator while using the genetic estimation to improve the behaviour of the scheme by modifying the set of external compensators. The closed-loop is proved to be stable under weak conditions on the population evolution, which is only required to be bounded at all time, in comparison with previous works where the evolution of the chromosomes influenced the stability properties being difficult or even impossible to guarantee. Moreover, the proposed approach is flexible enough to be integrated with a variety of genetic estimation algorithms proposed in the literature.

\section{APPENDIX I}

\section{Proof of Theorem 1}

Proof of Theorem 1. The proof is organised into different steps according to Section 4.

\section{1.- Proof of the boundedness of $r_{1}$.}

Consider the transfer function

$$
r_{1}=\hat{D}_{p k} C r=\frac{\hat{D}_{p k}(z) C_{n}(z)}{C_{d}(z)} r
$$

where $r$ denotes the reference input which is bounded by Assumption 4. Therefore there exists $\Gamma>0$ such that $\|r\| \leq \Gamma$. The state-space discrete-time representation of (AI.1) is:

$$
\begin{aligned}
& x_{k+1}=A_{1} x_{k}+B r_{1} r_{k} \\
& r_{1 k}=C_{1}^{T} x_{k}+D_{1} r_{k}
\end{aligned}
$$

with $\left(\mathrm{A}_{1}, \mathrm{~B}_{1}(k), \mathrm{C}_{1}(k), D_{1}(k)\right)$ where $\mathrm{A}==_{1}$ is a constant strictly stable matrix whose eigenvalues are the constant strictly stable zeroes of $C_{d}(z)$ from Assumption 5 [22]. However, $r_{1}$ might still diverge due to presence of the timevarying polynomial operator $\hat{D}_{p k}$ [23]. The solution to (AI.2) is: 
$x_{k}=\mathrm{A}_{1}{ }^{k} x_{0}+\sum_{j=0}^{k-1} \mathrm{~A}_{1}{ }^{k-j-1} \mathrm{~B}_{1}(j) r_{j}$

Upon Assumption 3 holds, there is $b>0$, such that

$\left\|\mathrm{B}_{1}(k)\right\|,\left\|\mathrm{C}_{1}(k)\right\|,\left\|D_{1}(k)\right\|<b \quad \forall k \geq 0$

Moreover, there are constants $C>1$ and $0<\rho<1$ such that $\left\|\mathrm{A}_{1}\right\|^{k} \leq C \rho^{k}$ since $\mathrm{A}_{1}$ is strictly stable. Hence, (AI.3) can be upper-bounded as:

$\left\|x_{k}\right\| \leq C \rho^{k}\left\|x_{0}\right\|+b \Gamma C \sum_{j=0}^{k-1} \rho^{k-j-1}$

or, equivalently:

$\left\|x_{k}\right\| \leq C \rho^{k}\left\|x_{0}\right\|+b \Gamma C \frac{1-\rho^{k}}{1-\rho}<\infty \quad \forall k \geq 0$

provided $\left\|x_{0}\right\|<\infty$, since $0<\rho<1$ and $\rho^{k} \rightarrow 0$ as $k \rightarrow \infty$.

In conclusion, $\left\|x_{k}\right\|<\infty, \forall k \geq 0$ and, hence,

$\left\|r_{1 k}\right\| \leq\left\|C^{T}\right\|\left\|x_{k}\right\|+\left\|D_{1}\right\|\left\|r_{k}\right\| \leq b\left\|x_{k}\right\|+b \Gamma<\infty$

Finally proving in the boundedness of $r_{1}$.

2.- Proof of the boundedness of $r_{2}$.

The relation between $r_{2}$ and the reference signal $r$ is:

$r_{2}=\hat{N}_{p k} C r=\frac{\hat{N}_{p k}(z) C_{n}(z)}{C_{d}(z)} r$

We consider the state-space description of the above system given by $\left(\mathrm{A}_{2}, \mathrm{~B}_{2}(k), \mathrm{C}_{2}(k), D_{2}(k)\right)$. As a result of the structure of the state-space realization being the same as in the previous item and the properties of the estimation algorithm still hold, the boundedness of $r_{2 k}$ is obtained by repeating the same steps as in the first point. Thus, we have already proved that the signals $r_{1}, r_{2}$ are both bounded.

\section{3.- Representation of the closed-loop system as a system with exogenous inputs}

Thus, we consider the control system with external signals represented in Fig. (5). The output is then computed to be:

$y=\frac{N_{p} S}{D_{p} S+N_{p} R} r_{2}+\frac{N_{p} R}{D_{p} S+N_{p} R} r_{1}$

Since Assumption 2 holds and $r_{1}, r_{2}$ are bounded signals from the previous steps, the output of the above LTI system is also bounded. Thus, the stability of the discrete-time closedloop system is finally proved. Hence, provided the stability of the discrete-time system and considering that there is no finite escape time since the plant is linear, it can be concluded from [22] the boundedness of the continuous-time original system.
Furthermore, since Steps 1 and 2 below hold for any $\left\|x_{0}\right\|<\infty$, the stability is global, finally proving Theorem 1 .

\section{REFERENCES}

[1] J. H. Holland, Adaptation in Natural and Artificial Systems, University of Michigan Press, 1975.

[2] L. Moyne, L. Porter II and K. M. Passino, "Genetic adaptive observers”, Eng. Appl. Art. Int., vol. 8, no. 3, pp. 261-269, 1995.

[3] M. Jamshidi, R. A. Krohling, Leandro dos S. Coelho, and P. J. Fleming, Robust Control Systems with Genetic Algorithms, CRC Press, 2002.

[4] T. L. Seng, M. Khalid and R. Yusof, "Tuning of a neuro-fuzzy controller by genetic algorithm", IEEE Trans. Syst. Man Cybern., vol. 29, no. 2, pp. 226-236, 1999.

[5] Y. Lee and S. H. Zak, "Designing a genetic neural fuzzy antilockbrake-system controller", IEEE Trans. Evol. Comp., vol. 6, no. 2, pp. 198-211, 2002.

[6] J. Chen and D. C. Rine "Training fuzzy logic based software components by combining adaptation algorithms", Soft Com., vol. 2, pp. 4860, 1998.

[7] D. S. Pereira and J. O. P. Pinto, "Genetic algorithm based system identification and PID tuning for optimum adaptive control" in 2005 IEEE/ASME Conf. on Advanced Intelligent Mechatronics, 2005, pp. 801-806.

[8] M. L. Moore, J. T. Musacchio and K. M. Passino, "Genetic adaptive control for an inverted wedge: experiments and comparative analysis", Eng. Appl. Art. Int., vol. 14, no. 1, pp. 1-14, 2001.

[9] W. Zuo, "Multivariable adaptive control for a space station using genetic algorithms", IEE Proc. Cont. Part. D, vol. 142, no. 2, 1995.

[10] K. Kristinsson and G. A. Dumont, "System identification and control using genetic algorithms", IEEE Trans. Sys. Man Cybern., no. 5, pp. 1033-1046, 1992.

[11] M. A. Marra and B. L. Walcott, "Stability and optimality in genetic algorithm controllers", in IEEE Symposium on Intelligent Control, 1996, pp. 492-496.

[12] W. K. Lennon and K. M. Passino, "Genetic adaptive identification and control", Eng. Appl. Art. Int., vol. 12, no. 2, pp. 185-200, 1999.

[13] T. Kumon, T. Suzuki, M. Iwasaki, M. Matsuzaki, N. Matsui and S. Okuma, "System identification using a genetic algorithm and its application to internal adaptive model control", Elec. Eng. Japan, vol. 142 , no. 4, 2003.

[14] A. Ibeas and M. de la Sen, "Artificial intelligence and graph theory tools for describing switched linear control systems", App. Art. Int., vol. 20, no. 9, pp. 703-741, 2006.

[15] J. I. Hidalgo, M. Prieto, J. Lanchares, F. Tirado, B. de Andrés, S. Esteban and D. Rivera, "A method for model parameter identification using parallel genetic algorihms" in Lectures Notes in Computer Science, vol. 1697, Springer-Verlag, Berlin Heidelberg, 1999, pp. 291 298.

[16] R. Vilanova and I. Serra, "Realization of two-degree-of-freedom compensators", IEE Proc. Part D., vol. 144, no. 6, pp. 589- 596, 1997.

[17] R. Vilanova, I. Serra, C. Pedret and R. Moreno, "Reference processing in 2-DOF control: separation, independence or optimality" in American Control Conference,2006 pp. 5680-5685.

[18] A. Bilbao-Guillerna, M. de la Sen, S. Alonso-Quesada, "On a root locus-based analysis of the limiting zeros of plants of nominal order at most two under FROH-discretization", in American Control Conference 2005 pp. 1205-1207.

[19] W. W. Kwok and D. E. Davison, "Implementation of stabilizing control laws”, IEEE Cont. Sys. Mag., vol. 27, no. 1, pp. 55-60, 2007.

[20] M. Morari and E. Zafirou, Robust Process Control. Prentice-Hall International, 1989.

[21] Sánchez Peña, R. S, Sznaier, M., Robust Systems Theory and Applications, Wiley Interscience, 1998.

[22] K. J. Astrom and B. Wittenmark, Computer Controlled Systems : Theory and Design. Prentice-Hall, 1984.

[23] K. S. Tsakalis and P. A. Ioannou, Linear Time-Varying Systems: Control and Adaptation. Prentice-Hall, 1992. 